\title{
Gaining Perspective
}

\section{Sangeetha Bobba*}

MBBS (Monash) FRACGP DCH Cert SH \& FPA GradCert MH, Australia

*Corresponding author: Sangeetha Bobba, MBBS (Monash) FRACGP DCH Cert SH \& FPA GradCert MH, Australia, Tel: +61410947645; E-mail: sangeetha.bobba@gmail.com

Rec date: May 22, 2014; Acc date: Jun 10, 2014; Pub date: Jun 12, 2014

Copyright: $\odot 2014$ Bobba S. This is an open-access article distributed under the terms of the Creative Commons Attribution License, which permits unrestricted use, distribution, and reproduction in any medium, provided the original author and source are credited.

\section{Introduction}

Working and living in a foreign country or simply in a foreign environment relative to one's known workplace can be a life altering experience. Doctors often return from overseas working stints whether paid or voluntary with amazing stories and an altered perspective. Working and living in disadvantaged communities with a different culture and language can be daunting and extremely challenging. Simultaneously, it is incredibly professionally and personally rewarding.

\section{Experiences from medical work in disadvantaged communities}

As a final year medical student I undertook an elective at the Christian Medical College Hospital in Southern India in 2007. Being Indian myself and having visited India since childhood I was expecting a sense of familiarity. Fortunately I did not experience the explosive gastroenteritis that non-Indian students experienced but I was similarly unprepared for witnessing the stark contrast in the medical presentations and the health care provision compared to Australia. Patients were packed into tiny consultation rooms, lined the hospital corridors, filled all the beds, occupied floors where no bed or seat was available and waited for their turn in queues outside the main hospital doors.

Patients commonly presented late due to affordability and accessibility barriers hence, medical conditions at first presentation were florid requiring drastic treatment measures such as amputation for what initially started as simple wound infections requiring antibiotics. I distinctly remember a village farmer diagnosed with extensive chromoblastomycosis requiring a lower limb amputation to the knee fraught with anxiety regarding how he was going to support his family following the amputation. Whilst on the paediatric ward a five year old girl who was being treated for severe malnutrition was discharged prematurely as her parents were unable to pay the hospital bills.

During my elective in India I was astounded by the value Indian people place on medical care, their appreciation of doctors and how fortunate we are in Australia, despite our grumblings regarding the shortcomings of the Australian medicare system, to have a health care system that is accessible and affordable to all Australians.

Following this experience, I was inspired to work in an area of need within Australia. In my resident year, I undertook a Prevocational General Practice Placement Program term in an Aboriginal Medical Service in the Northern Territory (NT). I was surprised to experience culture shock within my own country when I initially started working in the NT. Those who have worked in the top end will understand when I say that it is very different to the rest of Australia! People living in the NT have a relaxed outlook on life. In many ways this outlook is beneficial, perhaps for their blood pressure but not as their treating doctor! Patients presented late, patient adherence to treatment and rates of returning for review were frustratingly low. Factors such as affordability, transport, drug and alcohol issues, domestic violence, education, cultural differences and the Aboriginal history play significant contributory roles in the current state of individual patient stories and Aboriginal health statistics.

By working in the Northern Territory I became aware of the complexity of the provision of healthcare for Aboriginal people. I realised that unless you live and work in an area it is very difficult to understand the situation in its entirety. Superficial judgements and health care system changes recommended from an external perspective are simply not adequate and may potentially be harmful.

Following the long hours of my intern and resident years, I sought again to work, live and experience a different community. In 2010, I travelled to rural Kenya where I spent some time volunteering in a Maasai Village. Only once on the plane heading to Kenya did I feel a sense of apprehension, of not knowing what to expect and what was expected of me. I ended up being the sole doctor leading a team of volunteer nurses in a small medical centre. The clinic was composed of three consulting rooms and a small treatment room with basic surgical equipment. The clinic had the capacity to dispense broad-spectrum antibiotics, anti-malarials and nutritional supplements. The nearest hospital was in Nairobi four hours away by unsealed sandy roads. As a junior doctor this was quite frightening! I had more responsibility than I ever had in the hospital system in Australia and no medical colleagues to ask for assistance if required.

My time in Kenya was invaluable. Professionally, I learnt how to assess and diagnose conditions without the luxury of pathology tests or imaging, how to utilise the finite resources that were available and how to lead a team effectively in a challenging environment. I was exposed to issues such as lack of sexual health education, poor HIV awareness and female genital mutilation (FGM) that I had never faced before. The most memorable, rewarding and hopefully most lasting endeavour I undertook was teaching the Maasai adults about child nutrition, infection prevention and wound care and conducting a class at the nearby high school on sexual health and first aid.

In treating the Maasai people, they in turn provided me with so much more. I really appreciated the meaning and importance of community. I lived with a Maasai family whilst in Kenya and being involved in the day-to-day activities such as drawing water from the well, going to the market, attending ceremonies and working in the medical centre and the school made me feel part of the community. The acceptance and welcome I received was extraordinary. The Maasai people also taught me about gratitude and appreciation. For people who had so little they were generous, optimistic, resilient and 
genuinely happy. It was inspiring to witness and changed my perspective on how to view life.

My experience in Kenya exposed me first-hand to the harsh reality that the majority of women in the world have low standing and limited power in society. Treating women who underwent FGM and being confronted with its existence was very difficult. Although FGM is now illegal in Kenya in some communities it still occurs. However, having spoken to numerous male and female elders and educated young people, the views on FGM are progressively changing.

\section{Reflections from medical work experiences in disadvantaged communities}

In innumerable ways working and volunteering in culturally diverse and often disadvantaged communities can be beneficial to both the medical professionals and the host communities. However, there are also negative impacts of working and volunteering overseas and in areas of need. Due to the type of work and one's own life situation, these stints are often relatively short. So it can be questioned how beneficial it is in the long term for these communities without the continuity of care that is required. The introduction of Western viewpoints, education and medical methods may be beneficial in some cases but may also impact negatively on the host nation's culture, religion and societal system. From the perspective of the medical professional, the overwhelming feeling that the need is so great and seemingly endless is inevitable.

From working in these disadvantaged communities I felt that they may benefit from certain policy implementations. Hygiene and infection control policies would play an imperative role in decreasing the rates of infection. Educating the community in addition to medical and para-medical staff regarding basic infection control measures would be very beneficial. In addition, a systematic patient record keeping method and an ability to undertake audits would enable these communities to quickly identify respond to and contain communicable infections. A record keeping system would also allow for enhanced patient care and potential medical research which will ultimately benefit patients. As working in these communities can be professionally isolating, a policy of continuing medical education and avenues in providing support to medical and para-medical professionals in these communities is essential to patient outcome and retention of professionals in these communities.

On returning home from these experiences I felt an overwhelming sense of reverse culture shock. It was difficult to settle back into normal life and work, particularly following my time in Kenya. I felt uncomfortable and a sense of guilt with the ease of access and abundance of medical resources in Australia. I also initially felt a disconnect with my patients in Australia. I found it difficult to relate to their concerns regarding their depression or acne. This perhaps is why many doctors who have worked overseas in developing nations keep returning.

Working or volunteering in developing nations has its challenges. Possessing the awareness of the challenges, the needs and the potential negative impacts on the host community are essential. If undertaken with this insight visiting doctors can make a lasting beneficial impact. Ultimately, the statistics and numbers will always be too great however; altering the story at an individual level can mean a great difference for those people and their families. If receptive to the experience, the learning, self-awareness and humility gained is priceless. 